\title{
Introduction: A Special Issue on Cellular Biology of Protozoan Parasites
}

It is estimated that more than half of the human population, plus a much greater number of domestic and wild animals, suffer from parasitic infections. The magnitude of the problem can be illustrated by estimates of more than 100 million cases and 1 million deaths each year from malaria alone.

Parasites adapt to different environments and hosts, and it is therefore not surprising that they, many of them unicellular, have a number of peculiar structures not present in other living organisms. Investigations of these structures and their functional roles may provide information that can be used to selectively target the parasites without damaging the hosts. In addition, studies in parasites could provide insight into organisms where direct experimentation is not possible.

The objective of this issue is to present an overview of new instrumentation and methods and their applications to the microstructural analysis of the most relevant unicellular parasites as well as to report novel findings resulting from their use.

In the first article, Wanderley de Souza et al. offer an in depth description of the methods that have been applied to the study of Giardia lamblia, an organism responsible for a large number of cases of diarrhea throughout the world and of great biological interest because it represents one of the earliest lineages among eukaryotes. Giardia trophozoites possess two nuclei and lack many subcellular organelles characteristic of higher eukaryotes, such as peroxisomes and mitochondria. They also possess unique structures such as the adhesive disc and a very exuberant cytoskeleton. Giardia is an excellent model to provide insight into the minimal requirements for secretory transport, which in trophozoites is involved in their encystation.

Trichomonads are parasites that could affect the urogenital tract and cause important sexually transmitted diseases in humans and animals. Marlene Benchimol reviews the ultrastructure of these parasites with special emphasis on the process of cell division, the rich cytoskeleton, and peculiar organelles such as the hydrogenosome, which has been the subject of renewed enthusiasm among evolutionary biologists because of its relationship with the mitochondria. The biogenesis of the Golgi complex by an autonomous duplication mechanism was first described by Benchimol's group in trichomonads and is also reviewed.

Lawrence H. Bannister et al. report the 3-dimensional structure of the Plasmodium falciparum ring stage by reconstruction from serial sections and stereoscopic examination of tilted sections. P. falciparum is the most dangerous agent of human malaria, and the ring stage is the first of its erythrocytic stages. Evidence is also presented for a mechanism of exporting membrane from the parasite into the parasitophorous vacuole membrane, and beyond into the red blood cell, by means of double-membrane vesiclebased exocytosis. Felix A. Ruiz et al. report that P. falciparum merozoites possess electron-dense organelles rich in phosphorus and calcium, as detected by X-ray microanalysis of intact cells, which are similar to the acidocalcisomes of other Apicomplexans but of more irregular form. The presence of large amounts of polyphosphate and its changes during parasite development and chloroquine treatment are also reported.

Toxoplasma gondii is an obligate intracellular parasite of humans and animals that has emerged as a major opportunistic pathogen in people with AIDS and also as a cause of severe disease in the developing fetus. Marc-Jan Gubbel and Boris Striepen review recent advances made using fluorescent probes, such as constructs of green fluorescent protein with endogenous proteins, in the understanding of protein trafficking, organelle 
biogenesis, and cell division in T. gondii and in P. falciparum. The detection of the apicoplast, a relic plastic (or chloroplast) present in several Apicomplexan parasites, is of special interest. A technical section provides a collection of basic experimental protocols for fluorescent protein expression in T. gondii. Heide Schatten and Hans Ris describe a new specimen preparation technique, which allows three-dimensional imaging of thicksectioned internal cell structures by high-resolution low-voltage field emission scanning electron microscopy. The use of this method allowed them to visualize continuous structural interactions of the host cell membrane with T. gondii within the parasitophorous vacuole.

Cryptosporidium parvum, another Apicomplexan parasite, is the causative agent of cryptosporidiosis, an enteric disease of substantial medical and veterinary importance. C. parvum shows a number of unique features that differ from the rest of the Apicomplexans, among them the absence of an apicoplast. Franz Petry describes the structural analysis of the parasite using light and electron microscopy.

Neospora caninum is a recently described Apicomplexan parasite that has emerged as an important cause of abortion in cattle. Andrew Hemphill et al. focus on the use of tissue culture models as well as light and electron microscopy techniques for studies on $N$. caninum tachyzoites and bradyzoites, particularly the physical interactions between parasites and host cells.

The last section of this issue refers to studies in trypanosomatids, which belong to the order Kinetoplastida. These are causative agents of diseases of tremendous medical and economic importance in humans and animals, such as Chagas' disease caused by Trypanosoma cruzi, African sleeping sickness caused by the Trypanosoma brucei group of parasites, and leishmaniasis caused by Leishmania spp. In addition, the Phytomonas spp. cause diseases in plants. Trypanosomatids are a good example of parasites in which a number of peculiarities were first found in them and later discovered in other organisms, such as the existence of mitochondrial DNA, RNA editing, GPI-anchored proteins, and acidocalcisomes. Mark Field et al. describe how the application of several recent technical advances (transfection, inducible expression systems, and RNA interference), combined with genome sequencing efforts and microscopic analysis at the light and ultrastructural levels, have made T. brucei a model organism in cell biology. Celso Sant'Anna et al. report ultrastructural, cytochemical, and immunocytochemical studies on the biogenesis of reservosomes, an endocytic compartment present in T. cruzi, during reverse metacyclogenesis (conversion of trypomastigote to epimastigote stages) in vitro. Miranda et al. report ultrastructural and X-ray microanalysis studies on the identification of acidocalcisomes in the plant trypanosomatid Phytomonas françai. The presence of iron and a distinct morphology of these organelles are also described. Finally, Birgitta Rasmusson and Albert Descoteaux provide compelling evidence, using quantitative electron and confocal laser scanning microscopy approaches, that $L$. donovani promastigotes inhibit phagolysosome biogenesis in macrophages in a lipophosphoglycan-dependent manner. This inhibition correlates with an accumulation of periphagosomal F-actin, which may potentially form a physical barrier that prevents $L$. donovani promastigote-containing phagosomes from interacting with endocytic vacuoles.

Protozoan parasites have developed complicated structures in their adaptation to their hosts. In addition, the use of novel technical approaches is providing new targets for their elimination and unanticipated discoveries of general biological importance.

Roberto Docampo

Laboratory of Molecular Parasitology

Department of Pathobiology and Center for Zoonoses Research

University of Illinois at Urbana-Champaign 